\title{
A Character Condition for Quadruple Transitivity
}

\author{
S. Aldhafeeri and R. T. Curtis \\ School of Mathematics, University of Birmingham, Edgbaston, Birmingham B15 2TT, UK \\ Correspondence should be addressed to S. Aldhafeeri, saldhafeeri@yahoo.com
}

Received 9 May 2011; Accepted 22 June 2011

Academic Editor: Frank Werner

Copyright ( $) 2011$ S. Aldhafeeri and R. T. Curtis. This is an open access article distributed under the Creative Commons Attribution License, which permits unrestricted use, distribution, and reproduction in any medium, provided the original work is properly cited.

Let $G$ be a permutation group of degree $n$ viewed as a subgroup of the symmetric group $S \cong S_{n}$. We show that if the irreducible character of $S$ corresponding to the partition of $n$ into subsets of sizes $n-2$ and 2 , that is, to say the character often denoted by $x^{(n-2,2)}$, remains irreducible when restricted to $G$, then $n=4,5$ or 9 and $G \cong S_{3}, A_{5}$, or $P \Sigma L_{2}(8)$, respectively, or $G$ is 4-transitive.

\section{Introduction}

Let $G$ be a permutation group of degree $n$. The connection between the multiple transitivity of $G$ and the irreducibility of certain irreducible characters of of the symmetric group $S_{n}$ when restricted to $G$ goes back to Frobenius, and a proof of his classical result can be found in Tsuzuku [1]. In [2], Saxl strengthens this result, limiting the irreducibility hypothesis to the characters $X_{G}^{(n-k, k)}$, a special case of his theorem having been proved by Neumann [3]. In [4] Saxl uses the Classification of Finite Simple Groups to go much further. He obtains a complete list of pairs $(x, G)$ where $x$ is an irreducible character of the symmetric group $S_{n}$, and $G$ is a subgroup of $S_{n}$ such that the restriction of $X$ to $G$ remains irreducible.

The present paper is concerned with the case when $\chi_{G}^{(n-2,2)}$ is irreducible, proving that $G$ is 4-transitive except in three small cases which are worked out explicitly. As such, the result, which appears as Theorem 3.3, can be deduced from [2, 3, 5]. However, the approach used here is self-contained and elementary.

\section{Notation and Preliminary Results}

\subsection{Permutation Characters}

We start by recalling two fundamental results from the elementary permutation character theory. If a group $G$ permutes a set $X=\{1,2, \ldots, n\}$ then, we let $B=\left\{e_{1}, e_{2}, \ldots, e_{n}\right\}$ be 
a basis of an $n$-dimensional complex vector space, which becomes a $\mathbb{C} G$-module when we define $e_{i} v=e_{v(i)}$, for $v \in G$, and extend linearly. If $\pi_{X}$ denotes the character afforded by this representation and the permutation $v$ has cycle shape $1^{r} .2^{s} \ldots$ when written as a product of disjoint cycles, then

$$
\pi_{X}(v)=r=\left|\operatorname{Fix}_{X}(v)\right|
$$

where $\operatorname{Fix}_{X}(v)$ denotes the set of points of $X$ fixed by the permutation $v$. The subscript $X$ will be omitted when there is no fear of confusion. The standard inner product defined on the space of class functions when restricted to (generalized) characters becomes

$$
\langle x, \psi\rangle=\frac{1}{|G|} \sum_{g \in G} x(g) \overline{\psi(g)}
$$

where $\chi$ and $\psi$ are characters of the group $G$, and $\bar{\alpha}$ denotes the complex conjugate of the complex number $\alpha$. The trivial character of $G$ which takes the value 1 on every element of $G$ will be denoted by $1_{G}$. Then we have the following result; it is often referred to as Burnside's Lemma but is actually due to Cauchy and Frobenius.

Theorem 2.1. If $G$ is a permutation group acting on the set $X$ with permutation character $\pi_{X}$, then we have the following result:

$$
\left\langle\pi_{X}, 1_{G}\right\rangle=\operatorname{orb}(G, X),
$$

where $\operatorname{orb}(G, X)$ denotes the number of orbits when $G$ acts on $X$.

Proof. Firstly note that

$$
\left|\left\{(a, v) \mid\left\{\begin{array}{l}
a \in X \\
v \in G
\end{array}\right\}, a^{v}=a\right\}\right|=\sum_{a \in X}\left|\operatorname{Stab}_{G}(a)\right|=\sum_{v \in G}|\operatorname{Fix}(v)|
$$

where $\operatorname{Stab}_{G}(a)$ denotes the stabilizer in $G$ of $a$. So, if $G$ has orbits $O_{1}, O_{2}, \ldots, O_{m}$ when it acts on $X$, the Orbit-Stabilizer theorem implies that

$$
\sum_{a \in X}\left|\operatorname{Stab}_{G}(a)\right|=\sum_{i=1}^{m} \sum_{a_{i} \in O_{i}}\left|\operatorname{Stab}_{G}\left(a_{i}\right)\right|=\sum_{i=1}^{m}\left|O_{i}\right| \cdot\left|\operatorname{Stab}_{G}\left(b_{i}\right)\right|=m|G|
$$


where $b_{i}$ is a representative of the orbit $O_{i}$. Thus,

$$
\left\langle\pi_{X}, 1_{G}\right\rangle=\frac{1}{|G|} \sum_{v \in G}|\operatorname{Fix}(v)| \cdot 1=\frac{1}{|G|}|G| m=m .
$$

If the group $G$ acts on the set $X$ and on the set $Y$ with permutations characters $\pi_{X}$ and $\pi_{Y}$, respectively, then $G$ also acts on the Cartesian product $X \times Y$ by defining

$$
(x, y)^{v}=\left(x^{v}, y^{v}\right)
$$

An ordered pair is only fixed by $v$ if both, its components are fixed, and so we see that $\left|\operatorname{Fix}_{X \times Y}(v)\right|=\left|\operatorname{Fix}_{X}(v)\right| \cdot\left|\operatorname{Fix}_{Y}(v)\right|$. Then we have,

Theorem 2.2. If the group $G$ acts on the sets $X$ and $Y$ with permutation characters $\pi_{X}$ and $\pi_{Y}$ respectively, then

$$
\left\langle\pi_{X}, \pi_{Y}\right\rangle=\operatorname{orb}(G, X \times Y)
$$

Proof.

$$
\begin{aligned}
\left\langle\pi_{X}, \pi_{Y}\right\rangle & =\frac{1}{|G|} \sum_{v \in G}\left|\operatorname{Fix}_{X}(v)\right| \cdot \overline{\left|\operatorname{Fix}_{Y}(v)\right|}=\frac{1}{|G|} \sum_{v \in G}\left|\operatorname{Fix}_{X}(v)\right| \cdot\left|\operatorname{Fix}_{Y}(v)\right| \\
& =\frac{1}{|G|} \sum_{v \in G}\left|\operatorname{Fix}_{X \times Y}(v)\right|=\operatorname{orb}(G, X \times Y) .
\end{aligned}
$$

Corollary 2.3. If $G$ acts transitively on $X$ and $a \in X$, then $\left\langle\pi_{X}, \pi_{Y}\right\rangle=\operatorname{orb}\left(G_{a}, Y\right)$, the number of orbits of the stabilizer of a point in $\mathrm{X}$ on $\mathrm{Y}$.

Corollary 2.4. If $G$ acts transitively on $X$ and $a \in X$, then $\left\langle\pi_{X}, \pi_{X}\right\rangle=\operatorname{orb}\left(G_{a}, X\right)$, the rank of the permutation action of $G$ on $X$.

In the case when $S \cong \mathrm{S}_{n}$, the symmetric group acting on an $n$-element set $\Omega=$ $\{1,2, \ldots, n\}$, we denote the associated permutation character by $\pi_{1}$. More generally $\pi_{k}$ will denote the permutation action of $S \cong \mathrm{S}_{n}$ on unordered $k$-element subsets. In particular, $\pi_{2}$ will denote the permutation character of the action of $S$ on $\Delta=\{\{a, b\} \mid a, b \in \Omega\}$, the set of unordered pairs of elements of $\Omega$ which we shall call duads. Thus, if $v \in S$ has cycle shape $1^{r} .2^{s} \ldots$, then

$$
\pi_{1}(v)=r, \quad \pi_{2}(v)=\left(\begin{array}{l}
r \\
2
\end{array}\right)+s .
$$

By Theorem 2.1 and its corollaries we see that

$$
\left\langle\pi_{1}, 1_{S}\right\rangle=\left\langle\pi_{2}, 1_{S}\right\rangle=1, \quad\left\langle\pi_{1}, \pi_{1}\right\rangle=2, \quad\left\langle\pi_{1}, \pi_{2}\right\rangle=2, \quad\left\langle\pi_{2}, \pi_{2}\right\rangle=3 \quad(\text { for } n>3),
$$


since the stabilizer of a point clearly has two orbits on duads, and the stabilizer of a duad has three orbits on duads, namely, the orbit containing just the fixed duad, $\delta$ say, the orbit containing duads which intersect $\delta$ in one point, and the orbit of duads disjoint from $\delta$. We thus have that

$$
\pi_{1}=1_{G}+\chi, \quad \pi_{2}=1_{G}+\chi+\psi
$$

where $X$ and $\psi$ are distinct irreducible characters of $S$ of degrees $n-1$ and $n(n-3) / 2$, respectively.

\subsection{The Irreducible Characters of the Symmetric Group $S_{n}$}

Definition 2.5. A partition $\lambda$ of the integer $n$ is a sequence $\lambda=\left(\lambda_{1}, \lambda_{2}, \ldots, \lambda_{k}\right)$ where $\lambda_{i} \geq \lambda_{i+1}$ for $i=1, \ldots, k-1$ and $\sum_{i=1}^{k} \lambda_{i}=n$.

The irreducible characters of $S_{n}$ are in one-to-one correspondence with the partitions of $n$ and we denote the character corresponding to $\lambda$ by $\chi^{\lambda}$. In this notation, the trivial character of $S_{n}$ corresponds to the partition of $n$ into a single subset of size $n$ and is thus denoted by $1_{S}=x^{(n)}$. The permutation character of degree $n$ is given by $\pi_{1}=x^{(n)}+x^{(n-1,1)}$. Indeed we have the general result.

Theorem 2.6. If $k \leq n / 2$ and $\pi_{k}$ denotes the permutation character of the action of $S_{n}$ on unordered $k$-subsets, then

$$
\pi_{k}=x^{(n)}+x^{(n-1,1)}+\cdots+x^{(n-k, k)}
$$

That this permutation character is a sum of irreducibles of the right degree is readily shown and a proof may be seen in James and Liebeck [6, page 344]. That these irreducibles are indeed those corresponding to the above partitions may be verified using the celebrated Murnaghan-Nakayama rule, see [7, page 79], which enables one to evaluate the character value of the irreducible $\chi \lambda$ on any conjugacy class of the symmetric group. We see that for $k \leq n / 2$ we have

$$
\chi^{(n-k, k)}=\pi_{k}-\pi_{k-1}
$$

and so, if $\sigma \in \mathrm{S}_{n}$, then

$$
x^{(n-k, k)}(\sigma)=\mid\{k \text {-subsets fixed by } \sigma\}|-|\{(k-1) \text {-subsets fixed by } \sigma\} \mid
$$

In particular, the degree of $x^{(n-k, k)}$ is given by

$$
x^{(n-k, k)}(1)=\left(\begin{array}{l}
n \\
k
\end{array}\right)-\left(\begin{array}{c}
n \\
k-1
\end{array}\right) .
$$


Applying (2.15) to a permutation $\sigma$ with cycle shape $1^{r} .2^{s} \ldots$, we have

$$
x^{(n-2,2)}(\sigma)=\left(\begin{array}{l}
r \\
2
\end{array}\right)+s-r=\frac{r(r-3)}{2}+s
$$

\subsection{Multiple Transitivity}

Definition 2.7. The group $G$ is said to act $k$-transitively on the set $X$ if, and only if, given $\left(x_{1}, x_{2}, \ldots, x_{k}\right)$ and $\left(y_{1}, y_{2}, \ldots, x_{k}\right)$, two ordered $k$-tuples of distinct points of $X$, there exists a permutation $\sigma \in G$ such that $x_{i}^{\sigma}=y_{i}$ for $i=1 \cdots k$.

Definition 2.8. The group $G$ is said to act $k$-homogeneously on the set $X$ if, and only if, given $A, B \subset X$ with $|A|=|B|=k$, there exists a permutation $\sigma \in G$ such that $A^{\sigma}=B$.

In other words, $G$ is $k$-homogeneous if it acts transitively on the $k$-subsets. Those fascinating groups which are $k$-homogeneous without being $k$-transitive have been investigated by Kantor, see [8, 9], and the Livingstone-Wagner theorem of [10] is a highly significant result in this area. In this paper, we shall be concerned with the cases $k=$ 2,3 , and 4 and thus with the action of subgroups of $S_{n}$ on unordered pairs of points, which we shall refer to as duads, and on triples and tetrads. We can immediately see that the following applies.

Lemma 2.9. A group which is 2-homogeneous without being 2-transitive must have odd order.

Proof. Suppose that $G$ has even order and acts 2-homogeneously on $X$. Let $\alpha=\left\{a_{1}, a_{2}\right\}$ and $\beta=\left\{b_{1}, b_{2}\right\}$ be two duads; we must produce a permutation which maps $a_{i}$ to $b_{i}$ for $i=1$ and 2 . By 2-homogeneity, there exists a $\sigma \in G$ such that $\alpha^{\sigma}=\beta$, and since $G$ is even and again using the 2-homogeneity, there exists an element $\tau \in G$ of order 2 which acts as $\tau=\left(\begin{array}{ll}b_{1} & b_{2}\end{array}\right) \cdots$. If $a_{1}^{\sigma}=b_{1}$, then we are done, otherwise $\sigma \tau$ will suffice.

\subsection{Restriction to a Subgroup of $S_{n}$}

In what follows, the subgroup $G$ will be a fixed subgroup of $S \cong \mathrm{S}_{n}$, and we shall denote the restriction of the character $X$ of $S$ to $G$ by

$$
\left.x\right|_{G}
$$

In order to simplify the notation, we shall denote

$$
\left.\pi_{1}\right|_{G} \text { by } \psi_{1},\left.\pi_{2}\right|_{G} \text { by } \psi_{2},\left.x^{(n-1,1)}\right|_{G} \text { by } \psi^{(n-1,1)} \text {, and }\left.\chi^{(n-2,2)}\right|_{G} \text { by } \psi^{(n-2,2)} \text {. }
$$

So we shall have

$$
\psi_{1}=1_{G}+\psi^{(n-1,1)}, \quad \psi_{2}=1_{G}+\psi^{(n-1,1)}+\psi^{(n-2,2)} .
$$


The restriction to $G$ of an irreducible character of $S$ may or may not remain irreducible. However, if $G \leq S \cong S_{n}$, then using Theorems 2.1 and 2.2 we have

(1) $\left\langle\psi_{1}, 1_{G}\right\rangle$ is the number of orbits of $G$ on points,

(2) $\left\langle\psi_{2}, 1_{G}\right\rangle$ is the number of orbits of $G$ on duads,

(3) $\left\langle\psi_{1}, \psi_{1}\right\rangle$ is the number of orbits of $G$ on $\Omega \times \Omega$,

(4) $\left\langle\psi_{1}, \psi_{2}\right\rangle$ is the number of orbits of $G$ on $\Omega \times \Delta$, and

(5) $\left\langle\psi_{2}, \psi_{2}\right\rangle$ is the number of orbits of $G$ on $\Delta \times \Delta$.

Note that if $\chi$ and $\psi$ are as in (2.12), then $\left.\chi\right|_{G}=\psi^{(n-1,1)}$ and $\left.\psi\right|_{G}=\psi^{(n-2,2)}$. We shall use their correct labels in what follows but shall not make use of the correspondence with partitions in our argument.

\subsection{General Preliminary Results}

A result which will be appealed to several times in what follows is the following.

Lemma 2.10. The only permutation which commutes with a transitive permutation group and which fixes a point is the identity. In particular, a transitive, abelian permutation group acts regularly.

Proof. Let $K$ be a transitive subgroup of the symmetric group $S_{n}$ acting on the set $\Omega$, and let the permutation $\sigma \in \mathrm{S}_{n}$ commute with $K$ and fix a point $a \in \Omega$. Then $F=\operatorname{Fix}(\sigma) \subset \Omega$ is invariant under the action of $K$ and is thus the empty set $\phi$ or $\Omega$. But $a \in F \neq \phi$, and so $F=\Omega$ and $\sigma=1$.

If $K$ is abelian and $\sigma \in K_{a}$, the stabilizer in $K$ of $a$, then $\sigma$ commutes with $K$ and fixes the point $a$. Thus, $\sigma=1$, from the above, and so $K_{a}=\langle 1\rangle$, and the transitive group $K$ acts regularly.

A well-known result about linear groups has been taken from Theorem 7.3 of Huppert [11, page 187].

Theorem 2.11. The general linear group $\mathrm{GL}_{m}\left(p^{f}\right)$ contains a cyclic subgroup $K$ of order $p^{m f}-1$. We have $C_{G}(K)=K$ and that $N_{G}(K) / K$ is cyclic of order $m$. (Such cycles of maximal possible length are referred to as Singer cycles.)

\section{Statement and Proof of Main Theorem}

Let $G \leq \mathrm{S}_{n}$. The transitivity of $G$ is related to the reducibility of certain irreducible characters of $S$ upon restriction to $G$ as follows.

Lemma 3.1. $G$ is doubly transitive $\Leftrightarrow \psi^{(n-1,1)} \in \operatorname{Irr}(G)$.

Proof. Follows immediately from Corollary 2.4.

Lemma 3.2. $G$ is 4-transitive, $n \geq 4 \Rightarrow \psi^{(n-1,1)}, \psi^{(n-2,2)} \in \operatorname{Irr}(G)$. 
Proof. If $G$ is 4-transitive, then it is certainly doubly transitive, and so $\psi^{(n-1,1)} \in \operatorname{Irr}(G)$. Moreover, if $\delta$ is a duad, then $G_{\delta}$ certainly has just three orbits on duads, as described towards the end of Section 2.1. Thus,

$$
\begin{aligned}
& 3=\left\langle\psi_{2}, \psi_{2}\right\rangle=\left\langle 1_{G}+\psi^{(n-1,1)}+\psi^{(n-2,2)}, 1_{G}+\psi^{(n-1,1)}+\psi^{(n-2,2)}\right\rangle \geq 2+\left\langle\psi^{(n-2,2)}, \psi^{(n-2,2)}\right\rangle, \\
& \text { and so }\left\langle\psi^{(n-2,2)}, \psi^{(n-2,2)}\right\rangle=1 \text { and } \psi^{(n-2,2)} \in \operatorname{Irr}(G) \text {. }
\end{aligned}
$$

It is thus natural to ask in what circumstances we can have $\psi^{(n-2,2)} \in \operatorname{Irr}(G)$ without $G$ being 4-transitive. Our main result is the following theorem which appeals to the OddOrder theorem of Feit and Thompson and uses part of an argument due to Kantor [8] but is otherwise self-contained and based on Aldhafeeri [12].

Theorem 3.3. Let $G$ be a subgroup of the symmetric group of degree $n$ with $n \geq 4$, thus $G \leq S \cong S_{n}$, and let $x^{(n-2,2)}$ represent the irreducible character of $S$ corresponding to the partition of $n$ into a pair and a subset of cardinality $n-2$. Then

$$
\left.x^{(n-2,2)}\right|_{G} \in \operatorname{Irr}(G)
$$

(i.e., the restriction of $x^{(n-2,2)}$ to $G$ is an irreducible representation of $G$ ) if, and only if,

(i) $n=4$ and $G \cong \mathrm{S}_{3}$,

(ii) $n=5$ and $G \cong \mathrm{A}_{5}$,

(iii) $n=9$ and $G \cong P \Sigma \mathrm{L}_{2}(8)$, or

(iv) $G$ is 4-transitive on $n$ letters.

Proof. Note that the character $X^{(n-2,2)}$ of $S_{n}$ only exists for $n \geq 4$, and so the restriction on $n$ is only required to make the theorem meaningful. In case (i), the subgroup $G$ is not transitive, but no restriction was placed on the transitivity of $G$.

We shall assume that $G$ is not 4-transitive and that $\psi^{(n-2,2)} \in \operatorname{Irr}(G)$. We have $\psi^{(n-2,2)}(1)=n(n-3) / 2 \geq 2$ for $n \geq 4$. Thus,

$$
\left\langle\psi_{1}, 1_{G}\right\rangle=\left\langle\psi_{2}, 1_{G}\right\rangle=1+\left\langle\psi^{(n-1,1)}, 1_{G}\right\rangle
$$

and so $\operatorname{orb}(G, \Omega)=\operatorname{orb}(G, \Delta)=k$, say. But if $\operatorname{orb}(G, \Omega)=k$ then $\operatorname{orb}(G, \Delta) \geq\left(\begin{array}{l}k \\ 2\end{array}\right)$, as we may choose a duad from each of two orbits in $\left(\begin{array}{l}k \\ 2\end{array}\right)$ ways. Now $\left(\begin{array}{l}k \\ 2\end{array}\right)>k$ for $k>3$, and if $k=3$, at least one of the three orbits must have more than one point in it (since $n \geq 4$ ), and so $\operatorname{orb}(G, \Delta) \geq\left(\begin{array}{l}3 \\ 2\end{array}\right)+1=4$. Thus, $k \leq 2$. If $k=2$ and both orbits are of cardinality greater than 1 then $\operatorname{orb}(G, \Delta) \geq 3$, a contradiction, so we must have one orbit of length 1 and the other of length $n-1$.

Now $\psi^{(n-2,2)}(1)=n(n-3) / 2>n-1=\psi^{(n-1,1)}(1)$ provided $n^{2}-5 n+2>0$, which holds for $n \geq 5$. So provided $n>4$, we have $\left\langle\psi^{(n-2,2)}, \psi^{(n-1,1)}\right\rangle=0$. Thus,

$$
\operatorname{orb}(G, \Omega \times \Omega)=\left\langle\psi_{1}, \psi_{1}\right\rangle=\left\langle\psi_{1}, \psi_{2}\right\rangle=\operatorname{orb}(G, \Omega \times \Delta)=m, \text { say. }
$$


Table 1

\begin{tabular}{lcccc}
\hline $\mathrm{A}_{4}$ & 12 & 4 & 3 & 3 \\
& $1^{4}$ & $2^{2}$ & 1.3 & 1.3 \\
\hline$\psi^{(2,2)}$ & 2 & 2 & -1 & -1 \\
& 1 & 1 & $\omega$ & $\bar{\omega}$ \\
& 1 & 1 & $\bar{\omega}$ & $\omega$ \\
\hline
\end{tabular}

Table 2

\begin{tabular}{lccccc}
\hline \multirow{2}{*}{$\mathrm{D}_{8}$} & 8 & 8 & 4 & 4 & 4 \\
& $1^{4}$ & $2^{2}$ & 4 & $2^{2}$ & $1^{2} .2$ \\
\hline$\psi^{(2,2)}$ & 2 & 2 & 0 & 0 & 2 \\
& 1 & 1 & 1 & 1 & 1 \\
& 1 & 1 & -1 & -1 & 1 \\
\hline
\end{tabular}

Table 3

\begin{tabular}{lccc}
\hline $\mathrm{S}_{3}$ & 6 & 3 & 2 \\
& $1^{4}$ & 1.3 & $1^{2} .2$ \\
\hline$\psi^{(2,2)}$ & 2 & -1 & 0 \\
$\psi_{1}$ & 4 & 1 & 2 \\
\hline
\end{tabular}

For this reason, we treat $n=4$ separately and consider the circumstances in which this 2dimensional representation of $S_{4}$ can remain irreducible when restricted to a subgroup $G$. Since all irreducible representations of abelian groups are linear, we must have that $G$ is nonabelian, and the only proper non-abelian subgroups of $\mathrm{S}_{4}$ are $\mathrm{A}_{4}, \mathrm{D}_{8}$, and $\mathrm{S}_{3}$, fixing a point. In the following diagram we show the restrictions of $x^{(2,2)}$ to subgroups of $S_{4}$ isomorphic to $\mathrm{A}_{4}, \mathrm{D}_{8}$, and $\mathrm{S}_{3}$, respectively. The top line in Tables 1, 2, and 3 gives the order of the centralizer of elements in the conjugacy class of that column, and the second row gives the cycle shape of elements in that class (viewed as elements in $S_{4}$ ). It is clear that in the $A_{4}$ and $D_{8}$ cases the restricted character is the sum of the two linear characters shown, but in the $S_{3}$ case the restricted character is indeed irreducible.

We may now assume that $n \geq 5$ and that (3.4) holds. Let us return to the case reached above where $G$ has two orbits on $\Omega$, of lengths 1 and $n-1$, respectively. In order to dismiss this case (for $n>4$ ), we may assume that $G \cong \mathrm{S}_{n-1}$, as a character which remains irreducible when restricted to a subgroup and certainly remains irreducible when restricted to a supergroup of that subgroup. Suppose the two orbits of $G$ are $\{a\}$ and $\left\{b_{1}, b_{2}, \ldots, b_{n-1}\right\}$. But now $\operatorname{orb}(G, \Omega \times$ $\Omega)=5$ with orbit representatives $\left\{(a, a),\left(a, b_{1}\right),\left(b_{1}, a\right),\left(b_{1}, b_{1}\right),\left(b_{1}, b_{2}\right)\right\}$. However, $\operatorname{orb}(G, \Omega \times$ $\Delta)=6$ with orbit representatives

$$
\left\{\left(a,\left\{a, b_{1}\right\}\right),\left(a,\left\{b_{1}, b_{2}\right\}\right),\left(b_{1},\left\{a, b_{1}\right\}\right),\left(b_{1},\left\{a, b_{2}\right\}\right),\left(b_{1},\left\{b_{1}, b_{2}\right\}\right),\left(b_{1},\left\{b_{2}, b_{3}\right\}\right)\right\} .
$$

(Note that when $n=4$ and $G \cong S_{3}$, we do indeed have $\left\langle\psi_{1}, \psi_{1}\right\rangle=5$ and that $\left\langle\psi_{1}, \psi_{2}\right\rangle=$ $\left\langle\psi_{1}, \psi_{1}+\psi^{(2,2)}\right\rangle=6$ ). However, if $n>4$, this contradicts (3.4), and so we must have that $G$ acts transitively on $\Omega$ and on $\Delta$.

Now let $\Omega=\{a, b, c, \ldots\}$. The transitivity of $G$ together with (3.4) imply that the stabilizer of a point, $G_{a}$, has the same number of orbits on points as it has on duads. As above, if $G_{a}$ has $k$ orbits on $\Omega$, then it has at least $\left(\begin{array}{l}k \\ 2\end{array}\right)$ orbits on duads, so we must have that 
$2 \leq k \leq 3$. If $k=3$, then at least one of the orbits of $G_{a}$ must have length greater than 1 (since $n \geq 5$ ), and so the number of orbits of $G_{a}$ on duads is at least 4 , a contradiction. Thus, $k=2$ and $G$ is doubly transitive. In particular, $\psi^{(n-1,1)}, \psi^{(n-2,2)} \in \operatorname{Irr}(G)$, and we have

$$
\operatorname{orb}(G, \Omega \times \Delta)=2, \quad \operatorname{orb}(G, \Delta \times \Delta)=3 .
$$

Since $G_{\delta}$, the stabilizer in $G$ of the duad $\delta=\{a, b\}$, has just two orbits on $\Omega$, these must be $\{a, b\}$ and $\{c, d, \ldots\}$, and so $G_{\delta}$ acts transitively on $\Omega \backslash\{a, b\}$. The transitivity of $G$ on duads thus implies that $G$ is transitive on triples. Moreover, by (3.6) $G_{\delta}$ has just three orbits on duads which must consist of $\{a, b\}$ itself, of length 1 , the $2(n-2)$ duads such as $\{a, c\}$ which intersect $\delta$ in one point, and the $\left(\begin{array}{c}n-2 \\ 2\end{array}\right)$ duads disjoint from $\delta$.

We now wish to show that $G$ acts triply transitively on $\Omega$. So let $\{a, b, c\}$ and $\{x, y, z\}$ be two triples of points in $\Omega$; we must produce a permutation $\pi \in G$ such that $x^{\pi}=a, y^{\pi}=$ $b, z^{\pi}=c$. Now by the transitivity on duads, there exists a $\sigma_{1} \in G$ with $\{x, y\}^{\sigma_{1}}=\{a, b\}$. So $\{x, z\}^{\sigma_{1}}$ is a duad intersecting $\delta$ in 1 point. But $G_{\delta}$ acts transitively on the set of $2(n-2)$ such duads, and so there exists a $\sigma_{2} \in G_{\delta}$ such that $\left\{x^{\sigma_{1}}, z^{\sigma_{1}}\right\}^{\sigma_{2}}=\{a, c\}=\{x, z\}^{\sigma_{1} \sigma_{2}}$ and $\{x, y\}^{\sigma_{1} \sigma_{2}}=\{a, b\}$. Thus, $x^{\sigma_{1} \sigma_{2}}=a, y^{\sigma_{1} \sigma_{2}}=b$ and $z^{\sigma_{1} \sigma_{2}}=c$ as required.

That $G$ acts transitively on tetrads, that is, that $G$ is 4-homogeneous, follows immediately from the fact that $G$ is transitive on duads, and $G_{\delta}$ is transitive on duads disjoint from $\delta$. We now investigate from the first principles the circumstances in which $G$ is not 4transitive.

Let $T=\{a, b, c, d\}$ denote a tetrad, then the $G_{T}$ denotes the (set) stabilizer of $T$ in $G$, and $G_{[T]}$ denotes the point-wise stabilizer of $T$. The factor group $F=G_{T} / G_{[T]}$ gives the action of $G_{T}$ on the four points of $T$ and is thus isomorphic to a subgroup of $S_{4}$. If $F \cong S_{4}$, then $G$ is 4-transitive, since any tetrad can be mapped into $T$, and then the four points $\{a, b, c, d\}$ may be rearranged as we wish by elements of $G_{T}$. We shall now show that if $F \varsubsetneqq \mathrm{S}_{4}$, then $F \cong \mathrm{A}_{4}$.

If $\delta_{1}$ and $\delta_{2}$ are two disjoint duads, then the transitivity of $G$ on duads together with the transitivity of $G_{\delta_{1}}$ on duads disjoint from $\delta_{1}$ show that

$$
|G|=\left(\begin{array}{l}
n \\
2
\end{array}\right)\left(\begin{array}{c}
n-2 \\
2
\end{array}\right)|L|=\frac{n(n-1)(n-2)(n-3)}{4}|L|,
$$

where $L$ denotes the stabilizer of $\delta_{1}$ and $\delta_{2}$. Then, $G_{a}$, the stabilizer of a point, has, order

$$
\left|G_{a}\right|=\frac{(n-1)(n-2)(n-3)}{4}|L|,
$$

which is visibly divisible by 3 , and so $G$ contains elements of order 3 which fix a point. Thus, the factor group $F$ contains elements of cycle shape 1.3. Now by the triple transitivity, we see that the point stabilizer $G_{a}$ acts double transitively on $\Omega \backslash\{a\}$ and thus has even order. So $G$ contains involutions of cycle shape $1^{r} \cdot 2^{s}$ where $r \geq 1$ and $s \geq 2$, for if $s=1$, then $G$ contains transpositions, and the double transitivity of $G$ will then force $G \cong S_{n}$. Thus, $F$ contains elements which act as $2^{2}$ on the four points of $T$. The only subgroups of $S_{4}$ which contain elements of order 3 and such involutions are $\mathrm{A}_{4}$ and $\mathrm{S}_{4}$.

Note further that if $G$ contains involutions which fix more than 1 point, then $F$ will contain transpositions; we shall then have $F \cong S_{4}$, and $G$ will be 4 -transitive. So we may 
assume that every involution of $G$ has just one fixed point. Moreover, no element of $G$ may contain a 4-cycle as we could conjugate this 4-cycle into the positions of $T$ and would again have $F \cong S_{4}$. Thus every nontrivial element in a Sylow 2-subgroup of $G$ has order 2 and so Sylow 2-subgroups of $G$ are elementary abelian.

We have seen that the 2-point stabilizer $G_{a b}$ in $G$ is of odd order,and, therefore, by the Feit-Thompson theorem, it is soluble. A minimal normal subgroup of a soluble group is an elementary abelian $p$-group, see, for example, Scott [13, page 74], where in this case $p$ is odd. If $K \leq G_{a b}$ is such a subgroup, then, since $G_{a b}$ acts transitively on the duads disjoint from $\{a, b\}, K$ must act transitively on $\Omega \backslash\{a, b\}$, otherwise duads in different orbits of $K$ could not be mapped to duads in the same orbit. Moreover, $K$ is abelian and so by Lemma $2.10 \mathrm{~K}$ acts regularly. Any other minimal normal subgroup $L$ would have to intersect $K$ trivially as the intersection will also be normal. But if $l \in L, k \in K$ then $[l, k]=l^{-1} k^{-1} l k \in L \cap K=\langle 1\rangle$, and so $l$ commutes with the transitive subgroup $K$. Thus $\langle L, K\rangle$ is abelian and transitive, and so regular. Thus, $\langle L, K\rangle=K=L$, and $K$ is unique. $|K|=p^{d}=n-2$.

We now investigate the structure of the point stabilizer $G_{a}$ and will in fact show that it has a regular normal subgroup which is an elementary abelian 2-group. At this point, our work is close to that of Kantor [9], and we include, part of his argument: let $\tau \in G^{\{a, b\}}$ be a permutation which interchanges $a$ and $b$; since $\tau^{2} \in G_{a b}$, a group of odd order, we may assume that $\tau$ is an involution. By the uniqueness of $K$ we see that $K^{\tau}=K$ and so $|\langle K, \tau\rangle|=$ $2|K|=2(n-2)$. Moreover, $\tau$ has a unique fixed point, and $K$ acts transitively on $\Omega \backslash\{a, b\}$, and so $\left|\tau^{K}\right|=n-2$. Thus, we have

$$
\tau^{K}=\left\{\tau_{1}=\tau, \tau_{2}, \ldots, \tau_{n-2}\right\}=K \tau
$$

where each $\tau_{i}$ is an involution interchanging $a$ and $b$. So $K=\left\{\tau_{1} \tau, \tau_{2} \tau, \ldots, \tau_{n-2} \tau\right\}$, and it is clear that conjugation by $\tau$ inverts every element of $K$. But now we can prove the following.

Lemma 3.4. No two involutions of $G$ which contain the same transposition can fix the same point, nor can they have a further transposition in common.

Proof. We may assume that the involutions $\sigma_{1}$ and $\sigma_{2}$ both contain the transposition $(a, b)$. Then both $\sigma_{1}$ and $\sigma_{2}$ invert every element of $K$, and so $\sigma_{1} \sigma_{2}$ commutes with every element of $K$, and fixes $a$ and $b$. If $\sigma_{1}$ and $\sigma_{2}$ either have a fixed point in common or have a further transposition in common, then $\sigma_{1} \sigma_{2}$ fixes a point and commutes with a transitive group (on $\Omega \backslash\{a, b\})$. Thus, $\sigma_{1} \sigma_{2}=1$, and so $\sigma_{1}=\sigma_{2}$.

Corollary 3.5. For any tetrad $T=\{a, b, c, d\}$ there is a unique involution acting as $(a b)(c d)$ on the points of $T$ and a unique Klein four-group acting on $T$.

Proof. We have seen that the factor group $F$ acting on the four points of $T$ is isomorphic to $\mathrm{A}_{4}$; by the Lemma the element $\pi_{1}$ acting as $\left(\begin{array}{lll}a & b\end{array}\right)\left(\begin{array}{ll}c & d\end{array}\right)$ is unique. But if we conjugate this element by the element $\pi_{2}$ which acts as $(a c)(b d)$, we wil obtain an involution acting as $\left(\begin{array}{ll}a & b\end{array}\right)\left(\begin{array}{ll}c & d\end{array}\right)$ on $T$ which can only be $\pi_{1}$. Thus, $\pi_{1}^{\pi_{2}}=\pi_{1}$ and $\pi_{1} \pi_{2}=\pi_{2} \pi_{1}=\pi_{3}$, the unique involution which acts as $\left(\begin{array}{lll}a & d\end{array}\right)\left(\begin{array}{ll}b & c\end{array}\right)$ on $T$.

We are now in a position to count the number of involutions in $G$ and, in particular, to count the number of involutions which commute with a given involution. From Lemma 3.4, 
we see that there is a unique involution fixing a given point and containing a given transposition; thus, the number of involutions fixing a given point is

$$
\left(\begin{array}{c}
n-1 \\
2
\end{array}\right) / \frac{(n-1)}{2}=n-2
$$

It is clear that commuting involutions must fix one another's set of fixed points, and so, since our involutions have just one fixed point, commuting involutions have the same fixed points. Now a given involution is in

$$
\left(\begin{array}{c}
\frac{n-1}{2} \\
2
\end{array}\right)=\frac{(n-1)(n-3)}{8}
$$

Klein four-group, and each pair of commuting involutions fixes $(n-1) / 4$ tetrads. Thus, the number of involutions commuting with a given involution is

$$
\frac{(n-1)(n-3)}{8} \times 2 \div \frac{(n-1)}{4}=n-3
$$

Thus the $(n-2)$ involutions which fix a given point all commute with one another and so must form the nontrivial elements of an elementary abelian 2-group which must be normal in the point stabilizer. Thus, $n=2^{m}+1$ for some $m \geq 2$, and $2^{m}-1=p^{d}$ for some $d$ where $p$ is an odd prime. We have the following.

Lemma 3.6. If $2^{m}=p^{d}+1$ where $p$ is prime, then $d=1$ and $m$ is prime.

Proof. If $p \equiv 1 \bmod 4$, the right-hand side is congruent to 2 modulo 4 which is a contradiction since $m \geq 2$, and so the left-hand side is congruent to 0 modulo 4 . So $p \equiv 3 \bmod 4$, and $d$ must be odd, since even $d$ would result in the left-hand side being again congruent to 2 modulo 4. But now

$$
2^{m}=p^{d}+1=(p+1) \underbrace{\left(p^{d-1}-\cdots-p+1\right)}_{d \text { terms }} .
$$

The underbracketed term is a sum of an odd number of odd integers and so is odd, which is a contradiction unless $d=1$. But then $2^{m}-1$ cannot be prime unless $m$ is prime.

The point stabilizing subgroup $G_{a}$ thus has the shape

$$
G_{a} \cong 2^{m}: P: H,
$$

where $P$ is a cyclic subgroup of order $p=2^{m}-1$ and $H$ normalizes $P$. If $P=\langle x\rangle$, then $x$ acts on the elementary abelian group of order $2^{m}$, which may be regarded as an $m$-dimensional vector space over $\mathbb{Z}_{2}$, as a cycle of maximal length—known as a Singer cycle. The subgroup $P: H$ is thus acting faithfully as a subgroup of the general linear group $\mathrm{GL}_{m}(2)$. We now 
appeal to Theorem 7.3 in Huppert [11, page 187], which for convenience we have included here as Theorem 2.11. For our purposes, we have $f=1$ and $p=2$; the Theorem says that our group $P: H$ is a subgroup of a Frobenius group of shape $p: m$, so either $H \cong C_{m}$ or $H$ is trivial as $m$ is prime. We thus have

$$
|G|=\left(2^{m}+1\right)\left(2^{m}\right)\left(2^{m}-1\right) k
$$

where $k=m$ or $1, m$ is prime, and $n=2^{m}+1$.

But $G$ is transitive on tetrads, and the stabilizer of a tetrad acts as $\mathrm{A}_{4}$ on the 4 points of the tetrad. Thus, $\left(\begin{array}{c}n \\ 4\end{array}\right) \times 12$ divides $|G|$, and we have

$$
n(n-1)(n-2) k=\frac{n(n-1)(n-2)(n-3)}{4 !} .12 . s,
$$

for some integer s; so $(n-3) / 2$ divides $k$. If $k=1$, we must have $n=5$, when $G$ is a triply transitive subgroup of $S_{5}$ of the order 5.4.3 $=60$. Thus $G \cong A_{5}$. If $k=m$, we have $2^{m-1}-1$ divides $m$, and since $m$ is prime we have $2^{m-1}=m+1$, and so $m=3$. In this case, $|G|=9.8 .7 .3$. We now construct a permutation group of this order and degree 9 and show that it is unique.

We shall take the set $\Omega$ to be $\Omega=\{a=\star, b=\infty, 0,1, \ldots, 6\}$, that is, to say the projective line $\mathrm{P}_{1}(7)$ supplemented by the symbol $\star$. Without loss of generality, we take $x=\left(\begin{array}{lllllll}0 & 1 & 2 & 3 & 4 & 5 & 6\end{array}\right)$ normalized within $G_{a}=G_{\star}$ by $y=\left(\begin{array}{lll}1 & 2 & 4\end{array}\right)\left(\begin{array}{lll}3 & 6 & 5\end{array}\right)$. We now seek an elementary abelian group $E$ of order 8 which is normalized by $\langle x, y\rangle \cong 7: 3$. Now one of the 7 involutions of $E$ must commute with $y$, and since it has cycle shape $1.2^{4}$, it must be one of

(i) $\sigma_{1}=(\infty \quad 0)\left(\begin{array}{llll}1 & 3\end{array}\right)(2 \quad 6)(4 \quad 5)$,

(ii) $\sigma_{2}=(\infty \quad 0)\left(\begin{array}{lll}1 & 5\end{array}\right)\left(\begin{array}{lll}2 & 3\end{array}\right)\left(\begin{array}{ll}4 & 6\end{array}\right)$,

(iii) $\sigma_{3}=(\infty \quad 0)\left(\begin{array}{lll}1 & 6\end{array}\right)\left(\begin{array}{lll}2 & 5\end{array}\right)\left(\begin{array}{ll}4 & 3\end{array}\right)$.

Both $\sigma_{1}^{\langle x, y\rangle}$ and $\sigma_{2}^{\langle x, y\rangle}$ are indeed elementary abelian groups of order 8; however, $\sigma_{3}^{x}$ does not commute with $\sigma_{3}$. Moreover, the the permutation $(\infty)(0)\left(\begin{array}{lll}1 & 6\end{array}\right)\left(\begin{array}{ll}2 & 5\end{array}\right)\left(\begin{array}{ll}3 & 4\end{array}\right)$ of the symmetric group $\mathrm{S}_{8}$, which negates the elements of $\mathbb{Z}_{7}$, normalizes $\langle x, y\rangle$ and interchanges $\sigma_{1}$ and $\sigma_{2}$. So without loss of generality, we may assume that $G_{\star}=\left\langle x, y, \sigma_{1}\right\rangle \cong 2^{3}: 7: 3$.

We now consider the number of Sylow 7 -subgroups which $G$ contains. The only permutation of $S_{9}$ outside $P$ which commutes with $P$ is the transposition $(\star \infty)$ which does not have the desired cycle shape (and, in any case, would generate $S_{9}$ ). Thus, $C_{G}(P)=P$. We know that $N_{G_{\star}}(P)=\langle x, y\rangle \cong 7: 3$ which has the index 9.8.7.3/7.3 = 72 in $G$. Now $72 \equiv 2$ modulo7, and so $N_{G}(P)$ must have the order 42 and contain an involution commuting with $y$ and inverting $x$. This element must have, cycle shape $1.2^{4}$ as usual, and so without loss of generality it is $z=(\star \infty)\left(\begin{array}{ll}1 & 6\end{array}\right)(25)(34)$. Since generators for $G$ were reached without loss of generality, and since the group $P \Sigma \mathrm{L}_{2}(8)$-which consists of the projective special linear group $\mathrm{PSL}_{2}(8)$ with the field automorphism adjoined-is well known to have the required properties, we must have

$$
\langle x, y, z\rangle \cong \mathrm{P} \Sigma \mathrm{L}_{2}(8) .
$$


Table 4: The projective plane $\mathrm{P}_{1}(8)$.

\begin{tabular}{lccccccccc}
\hline$\eta^{i}$ & $\infty$ & 0 & 1 & $\eta$ & $\eta^{2}$ & $\eta^{3}$ & $\eta^{4}$ & $\eta^{5}$ & $\eta^{6}$ \\
\hline $\mathrm{f}(\eta)$ & $\infty$ & 0 & 1 & $\eta$ & $\eta^{2}$ & $\eta+1$ & $\eta^{2}+\eta$ & $\eta^{2}+\eta+1$ & $\eta^{2}+1$ \\
$\Omega$ & $\star$ & $\infty$ & 0 & 1 & 2 & 3 & 4 & 5 & 6 \\
\hline
\end{tabular}

However, for completeness, we exhibit the identification as permutations of the projective line $\mathrm{P}_{1}(8)$. The field of order 8 is obtained by extending $\mathbb{Z}_{2}$ by a root of $\eta^{3}+\eta+1$. In Table 4 we display elements of $\mathrm{P}_{1}(8)$ both as powers of $\eta$ and as quadratic expressions in $\eta$. We also show the correspondence with elements of $\Omega$ as used above. Our generators now become

$$
\begin{gathered}
x: u \longmapsto \eta u, \\
y: u \longmapsto u^{2} \\
\sigma_{1}: u \longmapsto u+1, \\
z: u \longmapsto 1 / u .
\end{gathered}
$$

This completes the proof of the main theorem.

\section{References}

[1] T. Tsuzuku, "On multiple transitivity of permutation groups," Nagoya Mathematical Journal, vol. 18, pp. 93-109, 1961.

[2] J.Saxl, "Characters of multiply transitive permutation groups," Journal of Algebra, vol. 34, pp. 528-539, 1975.

[3] P. M. Neumann, "Generosity and characters of multiply transitive permutation groups," Proceedings of the London Mathematical Society, vol. 31, no. 4, pp. 457-481, 1975.

[4] J. Saxl, "The complex characters of the symmetric groups that remain irreducible in subgroups," Journal of Algebra, vol. 111, no. 1, pp. 210-219, 1987.

[5] J. Saxl, "Characters and generosity of permutation groups," Proceedings of the American Mathematical Society, vol. 47, pp. 73-76, 1975.

[6] G. James and M. Liebeck, Representations and Characters of Groups, Cambridge University Press, New York, NY, USA, 2nd edition, 2001.

[7] G. D. James, The Representation Theory of the Symmetric Groups, vol. 682 of Lecture Notes in Mathematics, Springer, Berlin, Germany, 1978.

[8] W. M. Kantor, “4-homogeneous groups,” Mathematische Zeitschrift, vol. 103, pp. 67-68, 1968.

[9] W. M. Kantor, “k-homogeneous groups," Mathematische Zeitschrift, vol. 124, pp. 261-265, 1972.

[10] D. Livingstone and A. Wagner, "Transitivity of finite permutation groups on unordered sets," Mathematische Zeitschrift, vol. 90, pp. 393-403, 1965.

[11] B. Huppert, Endliche Gruppen. I, Die Grundlehren der Mathematischen Wissenschaften, Springer, Berlin, Germany, 1967.

[12] S. Aldhafeeri, Characters of multiply transitive groups, Ph.D. thesis, University of Birmingham, 2009.

[13] W. R. Scott, Group Theory, Prentice-Hall, 1964. 


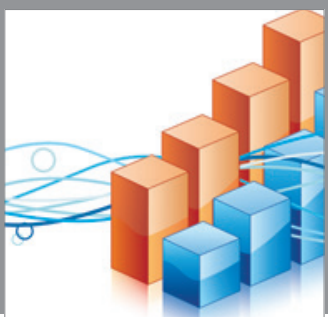

Advances in

Operations Research

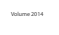

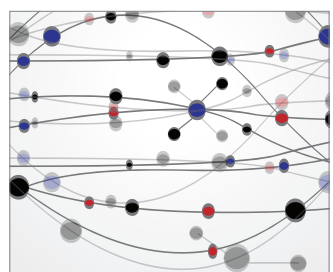

\section{The Scientific} World Journal
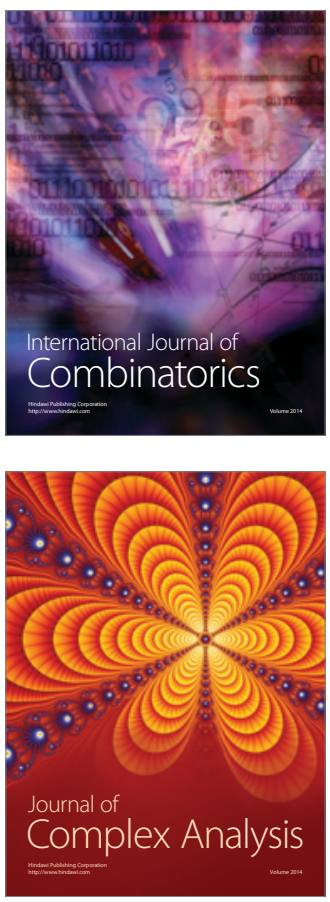

International Journal of

Mathematics and

Mathematical

Sciences
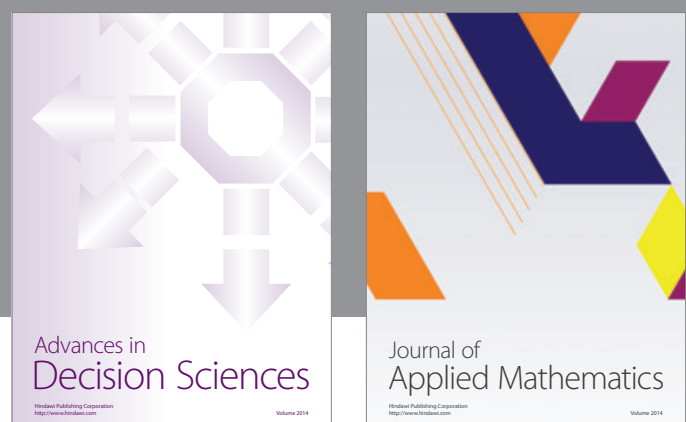

Journal of

Applied Mathematics
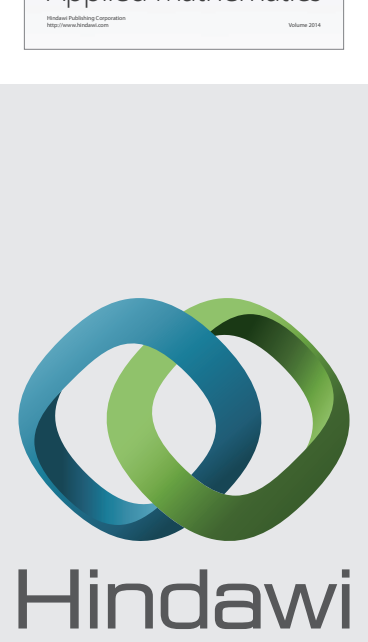

Submit your manuscripts at http://www.hindawi.com
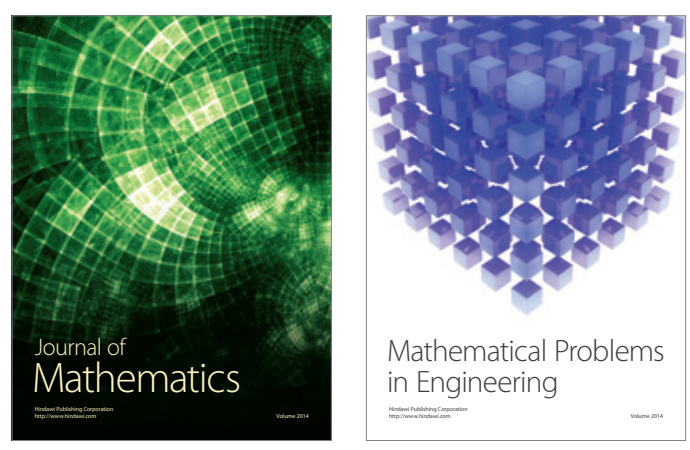

Mathematical Problems in Engineering
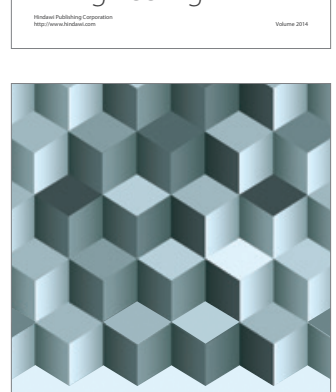

Journal of

Function Spaces
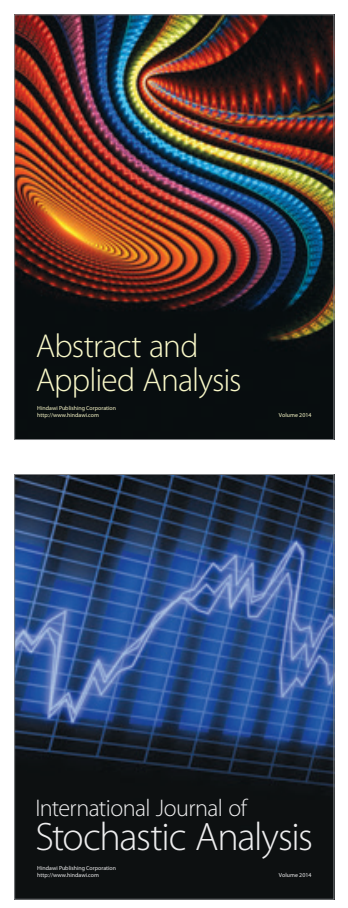

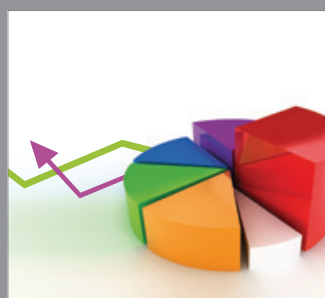

ournal of

Probability and Statistics

Promensencen
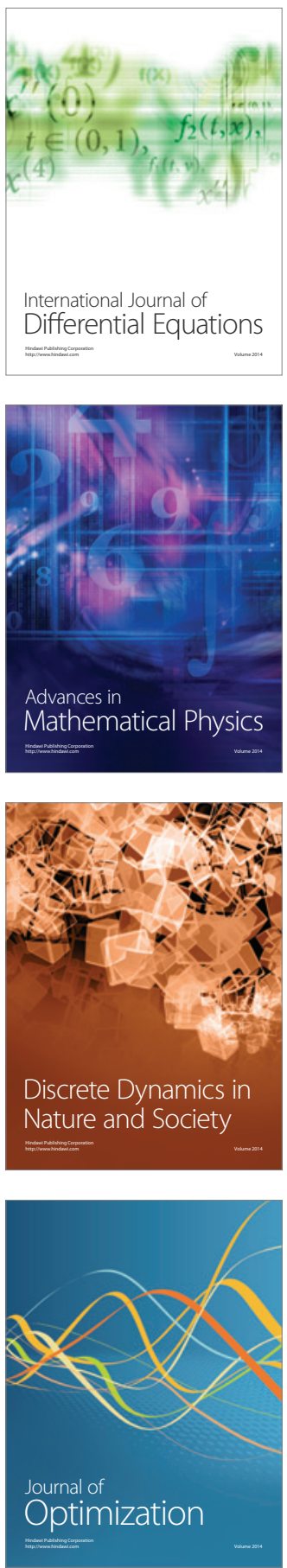